\title{
Moth Search Optimization for Optimal DERs Integration in Conjunction to OLTC Tap Operations in Distribution Systems
}

\author{
Pushpendra Singh ${ }^{\circledR}$, Student Member, IEEE, S. K. Bishnoi, and Nand K. Meena ${ }^{\circledR}$, Member, IEEE
}

\begin{abstract}
In this paper, a newly developed moth search optimization (MSO) technique is introduced to solve the complex distributed energy resources (DER) integration problems of distribution systems. In order to overcome some of the limitations observed in the standard variant of MSO, minor corrections are also suggested. On the other hand, a new optimization problem is formulated for optimal deployment of dispatchable distributed generations and shunt capacitors while simultaneously optimizing the tap positions of on-load tap-changing transformers, already deployed in grid substations. The objective of this work is to minimize the cost of annual energy loss and node voltage deviations over multiple load levels. The proposed model is implemented and solved for two benchmark test distribution networks of 33 and 118 buses. The suggested corrections are also validated by comparing the performance of the proposed approach with standard MSO and other available optimization methods. The simulation results show that the developed model optimally utilizes the existing distribution system resources and generates higher deployment benefits at lesser DER penetration as compared to the planning model which ignores these resources.
\end{abstract}

Index Terms-Distributed energy resources, distributed generation, distribution system, moth search optimization, on load tap changer, planning.

\section{INTRODUCTION}

D ISTRIBUTED generation (DG) has become an effective, reliable, and robust substitute for conventional power generation. The concept of small-sized power-generating units, at the vicinity of load end, has extended the working dimension of modern power distribution networks (PDNs) [1], [2]. The optimal integration of DGs may generate numerous benefits for utilities, customers, and owners [3], whereas, nonoptimal allocation of DGs may produce counterproductive solutions [4]. The socio-economic benefits of optimal DG allocation, in small- to large-scale distribution networks, have received the considerable attention from ecologists and economists across the globe.

Manuscript received September 1, 2018; revised January 20, 2019 and March 29, 2019; accepted April 11, 2019. (Corresponding author: Pushpendra Singh.) P. Singh is with the Department of Electrical Engineering, Govt. Women Engineering College, Ajmer, Rajasthan, and also with the Rajasthan Technical University, Kota, India (e-mail: pushpendragweca@gmail.com).

S. K. Bishnoi is with the Department of Electronics and Communications, Govt. Engineering College, Bikaner, India (e-mail: bishnoi_sk@yahoo.com).

N. K. Meena is with the School of Engineering and Applied Science, Aston University, Birmingham B4 7ET, U.K. (e-mail: nkmeena@ieee.org).

Digital Object Identifier 10.1109/JSYST.2019.2911534
The optimal integration of different DG types considering multiple/varying load levels may provide a compromising solution which can generate more benefits over variable loading conditions [5]-[7]. In the literature, the power loss minimization is considered as the primary objective while optimally allocating the DGs in PDNs along with several secondary objectives. These can be improvement of node voltage profile [5], [8], [9], power quality [10], voltage stability [8], [11]-[13], and reliability [10], [14], etc.

It may be observed that each DG type has its own profound impact on power system stability and economics. For example, lagging power factor DGs provide higher power loss reduction with improved voltage profile and stability in comparison to DGs operated at unity power factor (UPF) [11], [12], [15]-[17]. Generally, DGs are not allowed to participate in voltage control schemes rather operated at fixed power factor and considered as negative load. The frequent adjustment in generator excitation system in order to compensate required amount of reactive power in the system may cause overheating of the field winding [18]. Further, it may be observed that different types of voltage regulators (VRs) are already present in existing distribution systems to regulate the node voltages which may include shunt capacitors (SCs), on-load tap changer (OLTC), feeder VRs, etc. The participation of DGs in voltage regulation schemes may create coordination issues with existing VRs already present in the system.

In order to minimize some of the operational coordination issues and to improve the system performance, optimal allocation of different distributed energy resources (DERs) such as DGs and SCs is simultaneously obtained [6], [19]-[21]. A handful amount of work suggests the operational strategies like network reconfiguration while deploying DGs in distribution systems [6], [7], [22]. The simultaneous optimal allocation of DGs and OLTCs is also obtained in [23] for power loss minimization in distribution systems. Majority of existing research on DG integration are usually considering bare networks, assuming that there is no existing VR in PDNs. However, the traditional PDNs are already having various VRs to provide better voltage control which may limit the DG penetration. Therefore, it is suggested to use the existing VRs to control the node voltages in PDNs while operating DGs at fixed power factor. The consideration of existing VRs may also reduce the amount of DG penetration required to satisfy the node voltage limits constraints. 
The optimal allocation of DGs to determine optimal sites and sizes turns out to be a complex mixed integer, nonlinear, and nonconvex optimization problem. Thus, it requires fast and effective optimization methods to obtain the optimal solution out of enormous feasible solutions. In the literature, many analytical [17], [24] and Artificial Intelligence (AI) techniques [5], [6], [21], [25] have been suggested to optimally allocate the DGs in distribution systems. Some of the AI techniques may include genetic algorithm/particle swam optimization (GA/PSO) [13], teaching-learning-based optimization (TLBO) [6], [8], [25], improved elephant heard optimization (EHO) [11], water cycle optimization [21], etc. However, these methods show some inherent limitations when applied to real-life optimization problems such as high computation time and tendency to stuck in local optima [26]. For example, GA has unique information exchanging abilities by crossover and mutation operators; however, it has no memory and tendency of premature convergence. Although PSO has memory with simple operators, PSO and EHO also show premature convergence. The TLBO is parameter free and efficient to determine optimal solution but computationally inefficient due to two stages of teaching and learning. Therefore, powerful and robust optimization method with fast solution-searching abilities may be required to solve the optimal DG integration problems of distribution systems.

Recently, Wang introduced a new meta-heuristic optimization technique with fast convergence characteristics and better potential to explore global region, i.e., moth search optimization (MSO) [26]. It is a swarm intelligence-based technique inspired from the phototaxis and levy flying behavior of moth near its aim, usually fire. The flying behavior depends upon the distance of moth from the aim. The unique telling point of the method, over some of the existing methods, is its balanced exploitation and exploration, i.e., local and global searches. However, some limitations have been observed in standard variant of the method. As per the authors' knowledge, the technique has not been yet explored for optimal DER allocation problem of distribution systems.

In this paper, a newly developed MSO method is introduced to solve the complex optimal DER integration problem of distribution systems. Despite outperforming on benchmark functions, the method shows some limitations on real-life DER allocation problems of distribution systems. Therefore, some crucial corrections have also been suggested to overcome some of the limitations observed in its standard version [26]. Furthermore, a new DER allocation problem is proposed under existing voltageregulated framework of OLTC over multiple load levels aiming to minimize the cost of annual energy loss (AEL) and node voltage deviation. The proposed DER allocation model is implemented on two benchmark distribution systems, i.e., 33-bus and 118-bus, and then solved by modified MSO. First, the suggested corrections are validated, then the performance of proposed MSO and DER allocation model are compared with some of the existing optimization techniques and models, respectively. The modified MSO is observed to be efficient and fast to solve the DER allocation problems, whereas the consideration of existing OLTC in DG placement reduces the initial investment of DERs while providing improved performance of distribution systems.

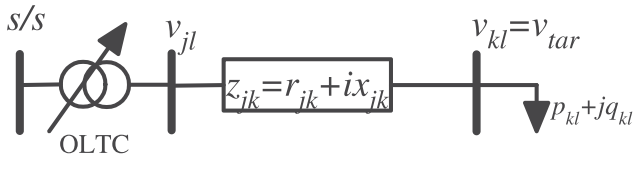

Fig. 1. Single-line diagram of OLTC and distribution feeder.

\section{Proposed Optimization Framework For Optimal DER INTEGRATION CONSIDERING EXISTING VOLTAGE REGULATION SCHEMES}

In this section, an optimization problem is developed, to optimally integrate different DERs, considering the existing OLTC operations simultaneously in PDNs over multiple load levels. In traditional PDNs, the node voltages are generally regulated by OLTCs associated with grid substation transformers. Many of the available DER allocation models [6]-[18], [20]-[24], [27] have ignored the presence of existing VRs in distribution systems and determined the optimal sites and sizes of DERs. However, ignoring existing resources may produce counterproductive results during system operations such as coordination issues, underutilization or overutilization of resources, and high initial investment cost of DERs. In this work, it is suggested to evaluate the effect of existing VRs, i.e., OLTC on optimal DER planning in PDNs. The voltage regulation by OLTC and objective function(s) is discussed in the following subsections.

\section{A. Voltage Regulation by OLTC}

Traditionally, the distribution systems were designed by considering unidirectional power flows in feeders. Accordingly, the various VRs were also dedicatedly employed in PDNs to regulate the node voltage profile, e.g., OLTCs, feeder VRs, SCs, etc. The OLTC is generally associated with a line-drop compensation (LDC) to estimate the voltage of target node. Based on the estimation and control algorithms, the OLTC tap position can be controlled manually or automatically.

The time-varying load demand has made the working of OLTC essential in order to regulate the bus voltages. In traditional architecture, it is designed with four-tap settings with an auxiliary winding which can sustain 2.5 percent spacing of full load voltage [28]. The design can maintain target bus voltage at nominal value with $\pm 5 \%$ voltage regulation capability. The single-line diagram of OLTC in the network is shown in Fig. 1. Mathematically, the tap setting of OLTC is expressed as in [28]

$$
t_{s}=\sqrt{\frac{\frac{\left|v_{k l}\right|}{\left|v_{j l}\right|}}{1-\frac{r_{j k} p_{k l}+x_{j k} q_{k l}}{\left|v_{j l}\right|\left|v_{k l}\right|}}}
$$

where $v_{j l}, v_{k l}, r_{j k}, x_{j k}, p_{k l}$, and $q_{k l}$ represent the sending end voltage, load or target bus voltage, resistance, reactance, real and reactive power flow of transmission line, respectively, for load level $l$. Practically, it may be observed that the OLTC taps are associated with certain number of turns of winding, and therefore, the taps are found to be discrete in nature. Sometimes, the tap setting suggested by (1) may not be an integer. In such 
cases, the nearest upper or lower tap can be selected depending upon the system requirements.

\section{B. Objective Function}

The AEL minimization while maintaining node voltages within specified limits has always been the primary concern of distribution network operators. Generally, it is observed from the literature that most of research works minimize the AEL in $\mathrm{kWh}$ or MWh which is not practiced. The annual energy demand and losses are time-varying, so does the cost of energy loss. Therefore, it would be beneficial to consider the cost of AEL minimization as one of the objective functions while integrating DERs in the system. The objective certainly affects the annual revenue of an utility. Similarly, the regulated node voltages can provide numerous benefits to utilities and consumers, and therefore, minimization of node voltage deviation has been considered as a second objective.

To minimize the cost of AEL and node voltage deviation simultaneously, a combined objective function is adopted in the proposed optimal DER integration problem under existing VR schemes. In this model, the problem is formulated as a single objective optimization problem and a penalty function-based approach is adopted to combine these two objective functions [29]-[31]. Here, the cost of AEL reduction is considered as the main objective, whereas voltage deviation is adopted as the penalty function. The combined objective or fitness function is expressed as

$$
\begin{aligned}
& \min F=F_{1}\left(t_{s}, p_{j l}, q_{j l}\right)\left[1+F_{2}\left(t_{s}, p_{j l}, q_{j l}\right)\right] \\
& F_{1}\left(t_{s}, p_{j l}, q_{j l}\right)=\sum_{l=1}^{n_{l}} C_{e, l} h_{l} \sum_{j=1}^{n} \sum_{k=1}^{n} \frac{r_{j k} \cos \left(\delta_{j l}-\delta_{k l}\right)}{v_{j l} v_{k l}} \\
& \left(p_{j l} p_{k l}+q_{j l} q_{k l}\right)+\frac{r_{j k} \sin \left(\delta_{j l}-\delta_{k l}\right)}{v_{j l} v_{k l}}\left(q_{j l} p_{k l}-p_{j l} q_{k l}\right) \\
& F_{2}\left(t_{s}, p_{j l}, q_{j l}\right)=\max <\max <\Delta v_{j l}>>\quad \forall j, l
\end{aligned}
$$

where $\Delta v_{j l}$ is the voltage penalty function expressed as

$$
\Delta v_{j l}= \begin{cases}\left|1-v_{j l}\right|, & \text { if } v_{\min } \leq v_{j l} \leq v_{\min } \\ 0, & \text { if } v_{\min } \leq v_{j l} \leq v_{\max } \\ \Omega, & \text { else }\end{cases}
$$

here, $\Omega$ is a large penalty factor with $\Omega>>F_{1}$, to avoid voltage violating solutions smoothly in optimization process. Equations (3) and (4) are expressed as the cost of AEL and node voltage deviation, respectively. Here, $n, n_{l}, h_{l}, C_{e, l}$, and $\delta_{j l}$ are representing the number of buses, load levels, hours in $l$ th load level, per-unit cost of energy purchase in $l$ th load level, and voltage angle of $j$ th bus during $l$ th load level, respectively. Further, $v_{\max }$, $v_{\min }$, and $v_{\operatorname{mins}}$ are the maximum, minimum, and minimum specified permissible limits of node voltages considered in per unit.
The objective function expressed in (2) is subjected to the following constraints:

$$
\begin{aligned}
& p_{j l}=v_{j l} \sum_{k=1}^{n} v_{k l} y_{j k} \cos \left(\theta_{j k}+\delta_{k l}-\delta_{j l}\right) \quad \forall j, l \\
& q_{j l}=-v_{j l} \sum_{k=1}^{n} v_{k l} y_{j k} \sin \left(\theta_{j k}+\delta_{k l}-\delta_{j l}\right) \quad \forall j, l \\
& v_{\min } \leq v_{j l} \leq v_{\max } \quad \forall j, l \\
& 0 \leq g_{j}^{\text {der }} \leq g_{\max }^{\operatorname{der}} \quad \forall j \\
& \sum_{j=1}^{n_{\text {der }}} g_{j}^{\text {der }} \leq \zeta_{p} \sum_{j=1}^{n} g_{j}^{d} \\
& i_{j k, l} \leq i_{j k}^{\max } \quad \forall j, k, l
\end{aligned}
$$

The constraints expressed in (6)-(11) are denoting the nodal real power balance, reactive power balance, voltage limits, DER penetration at single node, total DER penetration in the system, and thermal limits of feeders, respectively. Here, $y_{j k}, \theta_{j k}, i_{j k, l}$, and $i_{j k}^{\max }$ are the elements of Y-bus matrix, impedance angle, feeder current and its thermal limits of feeder connecting bus $j$ and $k$, for $l$ th load level, respectively. Moreover, $g_{j}^{\mathrm{der}}$, and $g_{j}^{d}$ are the suggested DER size and nominal load demand at node $j$, respectively. $g_{\max }^{\mathrm{der}}$, and $\zeta_{p}$ are the maximum allowed DER capacity at single node and load multiplying factor of peak load hours.

Considering the discrete system nodes, OLTC and SCs tap settings along with continuous DG sizes and other nonlinear functions and constraints, the proposed optimization model turns out to be a complex nonlinear, nonconvex, and mixed-integer optimization problem.

\section{MOTH SEARCH OPTIMIZATION}

In this section, the basics of MSO techniques are presented while suggesting minor corrections to overcome the limitations observed in them. The method was introduced by Gai-Ge Wang in 2016 [26]. MSO is a swarm intelligence-based optimization method with better exploitation and exploration capabilities as compared to other optimization methods. It is inspired by the self-organized and scattered behavior of moth swarm. The moths are attracted towards a light-emitting source which is their flying aim. The flights of a moth swarm are divided into two parts, namely, levy and straight flights, discussed in the following sections:

1) Lévy Flight: Lévy flight is a random walk where steplength follows heavy-tailed probability distribution. It is defined for one or more than one dimensions, the step sizes are in isotropic random directions, walk in space. The Lévy flights will be followed by the moths flying near to its aim, the light source. Here, the best moth is considered as the light source. The position of $i$ th moth with levy flight motion is updated as

$$
\begin{aligned}
z_{i}^{t+1} & =z_{i}^{t}+\gamma L(s) \\
\gamma & =\frac{S_{\max }}{t^{2}}
\end{aligned}
$$


here, $z_{i}^{t}$ and $z_{i}^{t+1}$ are representing the current and updated positions of $i$ th moth in $t$ and $(t+1)$ th generations, respectively. $L(s), \gamma$, and $S_{\max }$ are step-size drawn from Lévy flights, scale factor, and maximum walk step [26], respectively. The Levy distribution, $L(s) \sim|s|^{-\beta}$, is expressed as

$$
L(s)=\frac{(\beta-1) \Gamma(\beta-1) \sin \frac{\pi(\beta-1)}{2}}{\pi s^{\beta}}
$$

where $1<\beta \leq 3$, and $\Gamma(x)$ is the gamma function with $s>0$. The moth fly pattern is matching with Levy flight movement pattern with $\beta \approx 1.5$.

2) Straight Flight: It can be observed that certain moths which are far away from the light source will fly straight toward the aim in a line and mathematically expressed for $i$ th moth as

$$
z_{i}^{t+1}=\lambda\left(z_{i}^{t}+\phi\left(z_{\text {best }}^{t}-z_{i}^{t}\right)\right) .
$$

Sometimes, it is found that some moths cross their aim or light source. In standard MSO [26], the possibility of such moths is suggested to be $50 \%$. The position of such an $i$ th moth is updated as

$$
z_{i}^{t+1}=\lambda\left(z_{i}^{t}+\frac{1}{\phi}\left(z_{\text {best }}^{t}-z_{i}^{t}\right)\right)
$$

where $z_{\text {best }}^{t}$ is the position of the best moth in $t$ th generation and $\phi$ is golden ration, calculated as $\frac{\sqrt{5}-1}{2}$. Similarly, $\lambda$ is a scaling factor, chosen randomly and used to increase the diversity of algorithm. In straight flight, the position of a moth can be updated either by (15) or (16). Such moths are randomly selected by generating a random number, rand. If rand $>0.5$, the position of moth, in straight flight, is updated by (15), otherwise by (16).

It has been analyzed that the standard variant of MSO outperforms for benchmark functions and some of the engineering optimization problems as compared to artificial bee colony algorithm (ABC), biogeography-based optimization (BBO), differential evolution (DE), PSO, and stud GA (SGA). It is also observed that the standard MSO mostly converges to suboptimal solution when applied to complex optimization problems. The possible reason is unguided update in straight flights of moths expressed in (15) and (16). In these operators, the scale factor $\lambda$ is scaling both current, $z_{i}^{t}$, and the distance between light source and moth $\left(z_{\text {best }}^{t}-z_{i}^{t}\right)$, which results into fluctuating position of the moth in each iteration. Sometimes, the moth may also mislead from its previous position. In order to overcome the limitation, a random walk is suggested in straight flights of moth near to its current position. The suggested correction will help the moth to update its position with respect to its current and best moth's positions simultaneously. The modified equations (15) and (16) for straight flight are present in (17) and (18), respectively.

$$
\begin{aligned}
& z_{i}^{t+1}=z_{i}^{t}+\phi\left(z_{\text {best }}^{t}-z_{i}^{t}\right) \lambda \\
& z_{i}^{t+1}=z_{i}^{t}+\frac{1}{\phi}\left(z_{\text {best }}^{t}-z_{i}^{t}\right) \lambda .
\end{aligned}
$$

3) Correction Algorithm: It has been observed that some infeasible individuals are generated during optimization or position update, i.e., out of prescribed limits. In order to bring such

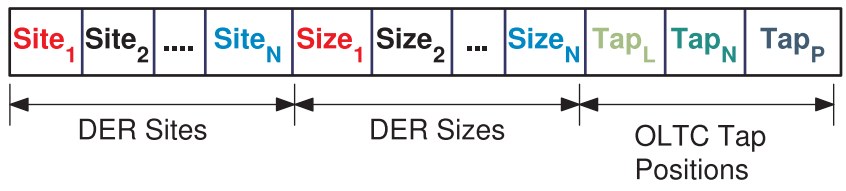

Fig. 2. The structure of individual used in the proposed MSO for DER integration in conjunction to OLTC tap control.

individuals within the predefined search boundaries, a correction algorithm may be needed. The following correction is suggested for infeasible individuals:

$$
z_{i}^{t+1}=L_{b}+\operatorname{rand} *\left(U_{b}-L_{b}\right) .
$$

Here, $L_{b}$ and $U_{b}$ are representing lower and upper limits of a variable, in an individual, at $b$ th position, as shown in Fig. 2.

\section{PROPOSED MSO FOR OPTIMAL DERS AlLOCATION}

This section describes the MSO technique adopted to solve optimal DER integration problems of PDNs. The structure of an individual, i.e., moth, is shown in Fig. 2. It contains the information of siting and sizing of DERs along with OLTC tap positions over different load levels.

The basic steps of the proposed MSO to solve the DER integration problems are presented below:

Step 1: Initialize the values of algorithm parameters such as $\beta$, $\phi, S_{\max }$, and maximum iterations.

Step 2: Generate initial but random populations of moth swam, " $P$."

Step 3: Calculate the fitness value, i.e., (2) of each moth using power flow calculation.

Step 4: Sort the population with respect to obtained fitness value.

Step 5: Update the upper half population of sorted individuals using (12).

Step 6: To update the lower half population of individuals, generate a random number for each remaining individual, rand. If rand $>0.5$, update the moths using (17), otherwise by (18)

Step 7: Apply the correction algorithm for infeasible individuals.

Step 8: Update the best, if a better individual is found.

Step 9: Repeat steps 3-8 till maximum iteration or stopping criteria is attained.

The flowchart of the proposed approach is presented in Fig. 3.

\section{CAse Study}

In this section, simulation results and case studies have been presented. The proposed optimization problem for optimal deployment of dispatchable DERs, developed in Section II, is solved by using modified MSO presented in Section IV. The optimal DER integration problems of two benchmark systems have been solved, namely, 33 and 118 buses [32], [33]. In practice, there may be many challenges to deploy DGs at some buses and depend on many factors such as availability of desired land, security, and technical ability of the buses and feeders to 


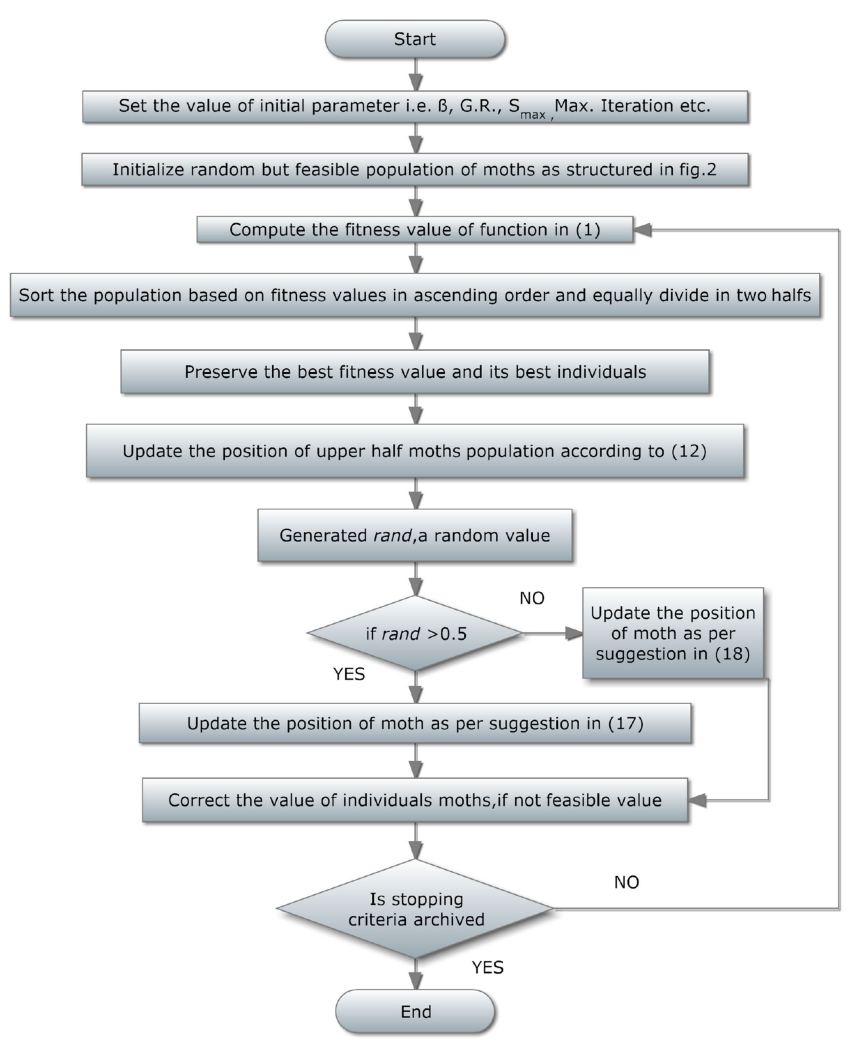

Fig. 3. Flowchart of the proposed MSO.

accommodate a size. The deployment of renewable DGs is further depending on availability of energy resources such as wind speed, solar irradiation, water, and waves. In such a situation, the optimization model can be applied to available potential nodes. In this work, all the nodes are assumed to be equally potential for DER installation. The dispatchable DGs are considered, and operated at UPF without participating in voltage regulations.

In order to validate the effectiveness of suggested improvements in standard MSO, a common DG integration problem for power loss minimization in 33-bus test distribution system is solved while keeping the OLTC tap at nominal position. After validation, the approach is applied to solve the proposed optimal DER integration problems in conjunction with OLTC tap operations. The proposed optimization problem is formulated in MATLAB platform and simulated on a Intel Core i5-6200U CPU@2.30GHz processor with 8GB RAM.

\section{A. Validation of Suggested Corrections in MSO for Optimal DER Allocation and Discussions}

To demonstrate the significance of suggested corrections in standard MSO and its ability to solve DER allocation problems of distribution systems, a simple DG integration problem of benchmark 33-bus distribution system for power loss minimization is formulated. The problem is solved by standard MSO [26] and MSO with suggested corrections called as corrected MSO (CMSO). The population size and maximum number of iterations considered for both the approaches are 50 and 100, respectively. Table I shows the comparison of simulation results
TABLE I

PERFORMANCE COMPARISON OF MSO AND CMSO FOR 50 INDEPENDENT TRAILS

\begin{tabular}{llllll}
\hline Method & $\begin{array}{l}\text { Best } \\
\text { Fitness }\end{array}$ & $\begin{array}{l}\text { Worst } \\
\text { Fitness }\end{array}$ & $\begin{array}{l}\text { Mean } \\
\text { Fitness }\end{array}$ & $\begin{array}{l}\text { Stand } \\
\text { Devia- } \\
\text { tion }\end{array}$ & $\begin{array}{l}\text { CPU } \\
\text { time(s) }\end{array}$ \\
\hline MSO & 0.0736 & 0.0908 & 0.0824 & 0.00378 & 7.41 \\
CMSO & 0.0714 & 0.0833 & 0.0757 & 0.00303 & 7.64 \\
\hline
\end{tabular}
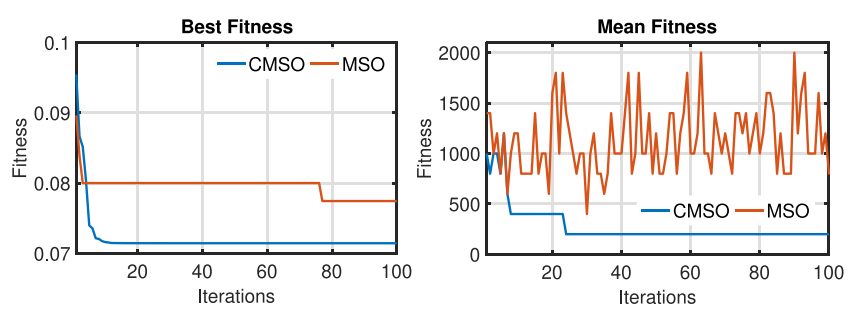

Fig. 4. Convergence characteristics of MSO and CMSO for power loss minimization in 33-bus distribution system.

TABLE II

COMPARISON OF SimULATION RESUlTS OBTAINED By CMSO and AVAILABle Optimization Methods

\begin{tabular}{llll}
\hline Method & $\begin{array}{l}\text { Optimal DG } \\
\text { Nodes }\end{array}$ & Size of DG (MW) & $\begin{array}{l}\text { Loss } \\
\text { (MW) }\end{array}$ \\
\hline TLBO [8] & $12,28,30$ & $1.183,1.191,1.186$ & 0.1246 \\
GA [13] & $11,29,30$ & $1.500,0.423,1.071$ & 0.1063 \\
PSO [13] & $08,13,32$ & $1.177,0.982,0.830$ & 0.1053 \\
GA/PSO [13] & $11,16,32$ & $0.925,0.863,1.200$ & 0.1034 \\
QOTLBO [8] & $13,26,30$ & $1.083,1.187,1.199$ & 0.1034 \\
IWO [9] & $14,18,32$ & $0.628,0.105,1.056$ & 0.0858 \\
LSF [16] & $18,25,33$ & $0.720,0.900,0.810$ & 0.0851 \\
IA [16] & $06,12,31$ & $0.900,0.900,0.720$ & 0.0811 \\
Analytical [17] & $06,16,25$ & $1.730,0.530,0.770$ & 0.0795 \\
ELF [16] & $13,24,30$ & $0.900,0.900,0.900$ & 0.0743 \\
DNPL-GA [5] & $14,24,30$ & $0.752,1.101,1.074$ & 0.0714 \\
CMSO & $14,24,30$ & $0.756,1.097,1.066$ & 0.0714 \\
\hline
\end{tabular}

obtained in 50 independent trials of MSO and CMSO. It summarizes the values of best fitness, worst fitness, mean fitness, standard deviation, and CPU time for MSO and CMSO. The comparison demonstrates that the suggested corrections are enhanced by the performance of MSO significantly to find optimal solution except the time which is slightly higher. The convergence characteristics of the methods are shown in Fig. 4. The best fitness characteristic shows the local trapping tendencies of standard MSO. It also shows that CMSO outperformed with suggested corrections. The mean fitness of standard MSO is not improving with the iterations/generations due to fluctuating guidance in straight flights.

Furthermore, to demonstrate the solution-finding ability of the proposed CMSO over some of the existing meta-heuristic and analytic optimization techniques, the power loss minimization of 33-bus distribution system obtained by CMSO and from available techniques is compared in Table II. The comparison is done with some well-established optimization methods such as TLBO, GA, PSO, GA/PSO, quasi-oppositional TLBO 
TABLE III

STUDY PARAMETERS [5]-[7], [22]

\begin{tabular}{lccc}
\hline Load level & $\begin{array}{c}\text { Load multiplying } \\
\text { factor, }(\zeta)\end{array}$ & $\begin{array}{c}\text { Load } \\
\text { duration }(\text { H) }\end{array}$ & $\begin{array}{c}\text { Energy price } \\
\text { (USD/MWh) }\end{array}$ \\
\hline Peak $(\mathrm{P})$ & 1.6 & 1500 & 120 \\
Normal $(\mathrm{N})$ & 1.0 & 5260 & 72 \\
Light $(\mathrm{L})$ & 0.5 & 2000 & 55 \\
\hline
\end{tabular}

(QOTLBO), invasive weed optimization (IWO), loss sensitivity factor (LSF), exhaustive load flow (ELF), improved analytical (IA), and dynamic node priority list-based GA (DNPL-GA). The comparison shows that CMSO has some better solution searching ability as compared to many of the existing well-established optimization methods. It has been observed that analytical methods such as LSF, ELF, and IA are fast in calculation but not efficient to search optimal solution. Mostly, some set of assumptions have been made in these methods.

\section{B. Simulation Results of Proposed OLTC-Based DER Integration}

After validating the proposed MSO, it is applied to determine optimal sites and sizes of different DERs while simultaneously optimizing the OLTC tap settings for cost of AEL minimization over multiple load levels. The information of load multiplying factor $(\zeta)$, load duration, and energy price for different load levels considered in this paper is adopted from [5] and also presented in Table III. The proposed DER integration problem framed in Section II is implemented and solved for two benchmark distribution systems. In this paper, it has been assumed that an OLTC is already employed in substation, i.e., node 1 for voltage regulation. In order to demonstrate the effectiveness of OLTC tap settings in optimal DER integration, the following cases have been framed and investigated in detail.

Case-I: Base case.

Case-II: Optimal integration of DGs only.

Case-III: Optimal integration of DGs in conjunction with OLTC taps settings.

Case-IV: Simultaneous optimal integration of DGs and SCs only.

Case-V: Optimal integration of DGs and SCs simultaneously in conjunction with OLTC taps settings.

The system-wise case studies are presented in the following sections:

1) Study System-I (33-Bus Radial Distributed Test System): It is a $12.66 \mathrm{kV}$ radial test distribution system with total real power demand of $3.715 \mathrm{MW}$, and reactive power demand of 2.300 MVAr [32]. The optimal DER integration problem presented in Section II is solved for the test cases framed in previous section aiming to determine optimal sites and sizes of different DERs in distribution systems. The optimal solutions obtained for these cases are compared with the same available in the literature and presented in Table IV. Table IV shows the optimal sites and sizes of DERs, DER penetration, power losses for different load levels, AEL, and cost. As can be observed from Case-II, the proposed MSO has the ability to provide most promising solution as compared to existing optimization methods such as HSA [22], FWA [7], and DNPL-GA [5]. These methods are unable to provide the required amount of DG penetration for this system. Now, the optimal tap settings of OLTC, and DG sites and sizes are simultaneously determined in Case-III for effective utilization of existing distribution system resources. The consideration of OLTC in conjunction with optimal DG placement provides more benefits in terms of higher power loss reduction at lesser DG penetration in comparison to Case-II. This investigation shows that the consideration of existing VRs may reduce the initial DER investment while offering more benefits as compared to the planning models where it was ignored.

In Case-IV, the optimal allocation of DGs and SCs is simultaneously determined. The capacitor banks of $100 \mathrm{kVAr}$ are considered in the entire study. The reactive power support provided by SCs drastically reduces the cost of AEL as compared to the case of DG only integration, as discussed in Case-II. On the other hand, the proposed MSO is generating better optimal solution in comparison to ITLBO [6] and DNPL-GA [5], due to its better solution searching ability. The ITLBO is unable to determine the required amount of DER penetration because the cost of energy for different load levels is not considered in [6]. The peak energy price promotes to higher DER penetration.

The OLTC tap settings, sites, and sizes of DGs and SCs are simultaneously optimized in Case-V. The simulation results show that the consideration of OLTC operations in optimal allocation of DERs provides higher power loss reduction even at lower DER penetration in comparison to Case-IV. It may also be observed that SCs are reducing the tap positions of OLTCs with reference to Case-III.

The proposed DER allocation problem shows that the simultaneous consideration of multiple DERs and VRs generates more techno-economic benefits for utilities, consumers, and DER owners. The consideration of VRs is helping the DERs to maintain the node voltages within limits at lesser DER penetration during peak load hours since the OLTC is moving its tap position in these hours. It may also be observed that simultaneous optimization of DER allocation and OLTC tap settings is providing better solution in comparison to coordinated control of OLTC presented in [5].

In order to validate the robustness of the proposed DER allocation model under voltage-regulated environment, it is also solved for a large-scale distribution system, as presented in the following section.

2) Study System-II(118-Bus Radial Distributed Test System): It is a large-scale radial test distribution system with total real and reactive power demands of $22.71 \mathrm{MW}$ and 17.04 MVAr, respectively [33]. The same test cases are investigated for this system and the simulation results are presented in Table V. In base case condition, the system shows poor node voltages during peak and nominal load levels. The optimal allocation of DGs is achieved in Case-II which improved the node voltage profile and reduced the cost of AEL significantly, as compared to base case. Now, the optimal allocation of DGs is obtained in conjunction with OLTC tap positions in Case-III. Consideration of OLTC reduces the cost of AEL at lesser DG penetration as compared 
TABLE IV

COMPARISON OF Simulation RESUlts ObTAINEd By CMSO AND OTHER OPTIMIZATION METHOdS FOR STUdy SySTEM-I

\begin{tabular}{|c|c|c|c|c|c|c|c|c|c|}
\hline Case & Method & $\begin{array}{l}\text { Optimal } \\
\text { [DG]/(SC) } \\
\text { nodes }\end{array}$ & $\begin{array}{l}\text { Optimal }[\mathrm{DG}] /(\mathrm{SC}) \\
\text { Sizes (MW/MVAr) }\end{array}$ & $\begin{array}{l}\text { OLTC } \\
\text { Tap }\end{array}$ & $\begin{array}{l}\text { DER } \\
\text { Pen.\% }\end{array}$ & $\begin{array}{l}\text { Power } \\
\text { Loss } \\
\text { (MW) }\end{array}$ & $V_{\min }$ & $\begin{array}{l}\text { AEL } \\
\text { (MWh) }\end{array}$ & $\begin{array}{l}\text { Cost of } \\
\text { AEL } \\
\text { (USD) }\end{array}$ \\
\hline \multirow[t]{3}{*}{ I } & \multirow[t]{3}{*}{-} & \multirow[t]{3}{*}{-} & \multirow[t]{3}{*}{ NA } & \multirow[t]{3}{*}{-} & \multirow[t]{3}{*}{00.00} & $0.5753^{P}$ & $0.85^{P}$ & \multirow[t]{3}{*}{2023} & \multirow[t]{3}{*}{185,480} \\
\hline & & & & & & $0.2027^{N}$ & $0.91^{N}$ & & \\
\hline & & & & & & $0.0471^{L}$ & $0.96^{L}$ & & \\
\hline \multirow{12}{*}{ II } & \multirow[t]{3}{*}{ HSA [22] } & \multirow[t]{3}{*}[17,18,33]{} & {$[0.911,0.194,1.612]^{P}$} & \multirow[t]{3}{*}{ NA } & \multirow[t]{3}{*}{38.86} & $0.2610^{P}$ & $0.94^{P}$ & \multirow[t]{3}{*}{946.99} & \multirow[t]{3}{*}{86,181} \\
\hline & & & {$[0.572,0.107,1.046]^{N}$} & & & $0.0968^{N}$ & $0.97^{N}$ & & \\
\hline & & & {$[0.178,0.130,0.503]^{L}$} & & & $0.0233^{L}$ & $0.98^{L}$ & & \\
\hline & \multirow[t]{3}{*}{ FWA [7] } & \multirow[t]{3}{*}[14,18,32]{} & {$[0.944,0.301,1.678]^{P}$} & \multirow[t]{3}{*}{ NA } & 41.81 & $0.2381^{P}$ & $0.95^{P}$ & 866.3 & 78,788 \\
\hline & & & {$[0.590,0.190,1.015]^{N}$} & & & $0.0887^{N}$ & $0.97^{N}$ & & \\
\hline & & & {$[0.295,0.095,0.507]^{L}$} & & & $0.0214^{L}$ & $0.98^{L}$ & & \\
\hline & DNPL-GA [5] & {$[14,24,30]$} & {$[0.842,1.201,1.246]^{P}$} & NA & 47.05 & $0.2210^{P}$ & $0.93^{P}$ & 742.05 & 68,753 \\
\hline & & & {$[0.754,1.100,1.071]^{N}$} & & & $0.0715^{N}$ & $0.97^{N}$ & & \\
\hline & & & {$[0.374,0.543,0.527]^{L}$} & & & $0.0173^{L}$ & $0.98^{L}$ & & \\
\hline & CMSO & {$[13,25,30]$} & {$[1.459,0.987,1.795]^{P}$} & NA & 60.65 & $0.1964^{P}$ & $0.95^{P}$ & 712.20 & 64,820 \\
\hline & & & {$[0.841,0.986,1.116]^{N}$} & & & $0.0727^{N}$ & $0.97^{N}$ & & \\
\hline & & & {$[0.416,0.476,0.514]^{L}$} & & & $0.0176^{L}$ & $0.98^{L}$ & & \\
\hline & DNPL-GA [5] & {$[14,24,30]$} & {$[0.869,1.062,1.168]^{P}$} & $3^{P}$ & 44.33 & $0.2087^{P}$ & $0.96^{P}$ & 711.41 & 65,659 \\
\hline & & & {$[0.763,1.062,0.865]^{N}$} & $2^{N}$ & & $0.0692^{N}$ & $0.99^{N}$ & & \\
\hline & & & {$[0.374,0.543,0.528]^{L}$} & $0^{L}$ & & $0.0173^{L}$ & $0.98^{L}$ & & \\
\hline III & CMSO & {$[13,25,30]$} & {$[1.249,0.976,1.474]^{P}$} & $3^{P}$ & 52.90 & $0.1801^{P}$ & $0.99^{P}$ & 666.72 & 37,992 \\
\hline & & & {$[0.819,0.972,1.011]^{N}$} & $2^{N}$ & & $0.0687^{N}$ & $0.99^{N}$ & & \\
\hline & & & {$[0.415,0.476,0.513]^{L}$} & $0^{L}$ & & $0.0176^{L}$ & $0.98^{L}$ & & \\
\hline & ITLBO [6] & {$[14,24,30]$} & {$[0.823,1.074,1.067]^{P}$} & NA & 49.91 & $0.0820^{P}$ & $0.95^{P}$ & 191.83 & 19,612 \\
\hline & & $(15,24,30)$ & {$[0.747,1.074,1.054]^{N}$} & & & $0.0119^{N}$ & $0.99^{N}$ & & \\
\hline & & & {$[0.374,0.540,0.517]^{L}$} & & & $0.0030^{L}$ & $1.00^{L}$ & & \\
\hline & & & $(0.300,0.600,1.200)^{P}$ & & & & & & \\
\hline & & & $(0.300,0.500,1.000)^{N}$ & & & & & & \\
\hline & & & $(0.200,0.300,0.500)^{L}$ & & & & & & \\
\hline & DNPL-GA [5] & {$[14,24,30]$} & {$[0.829,1.191,1.164]^{P}$} & NA & 58.66 & $0.0737^{P}$ & $0.95^{P}$ & 178.15 & 18,033 \\
\hline & & $(14,24,30)$ & {$[0.748,1.078,1.047]^{N}$} & & & $0.0118^{N}$ & $0.99^{N}$ & & \\
\hline IV & & & {$[0.373,0.538,0.522]^{L}$} & & & $0.0029^{L}$ & $1.00^{L}$ & & \\
\hline 10 & & & $(0.300,0.600,1.200)^{P}$ & & & & & & \\
\hline & & & $(0.400,0.500,1.000)^{N}$ & & & & & & \\
\hline & & & $(0.200,0.300,0.500)^{L}$ & & & & & & \\
\hline & CMSO & {$[11,24,31]$} & {$[1.517,1.358,0.872]^{P}$} & NA & 58.66 & $0.0592^{P}$ & $0.96^{P}$ & 177.40 & 16,906 \\
\hline & & $(6,12,30)$ & {$[0.994,1.099,0.783]^{N}$} & & & $0.0154^{N}$ & $0.97^{N}$ & & \\
\hline & & & {$[0.478,0.538,0.435]^{L}$} & & & $0.0038^{L}$ & $0.99^{L}$ & & \\
\hline & & & $(0.900,0.700,0.900)^{P}$ & & & & & & \\
\hline & & & $(0.500,0.400,0.800)^{N}$ & & & & & & \\
\hline & & & $(0.200,0.200,0.400)^{L}$ & & & & & & \\
\hline & DNPL-GA [5] & {$[13,24,30]$} & {$[0.848,1.177,1.143]^{P}$} & $3^{P}$ & 54.37 & $0.0696^{P}$ & $0.99^{P}$ & 172.73 & 17,350 \\
\hline & & $(14,24,30)$ & {$[0.779,1.073,1.035]^{N}$} & $0^{N}$ & & $0.0119^{N}$ & $0.99^{N}$ & & \\
\hline & & & {$[0.388,0.536,0.516]^{L}$} & $0^{L}$ & & $0.0029^{L}$ & $1.00^{L}$ & & \\
\hline & & & $(0.300,0.600,1.200)^{P}$ & & & & & & \\
\hline & & & $(0.300,0.500,1.000)^{N}$ & & & & & & \\
\hline $\mathrm{V}$ & & & $(0.200,0.300,0.500)^{L}$ & & & & & & \\
\hline 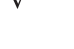 & CMSO & {$[10,24,29]$} & {$[1.132,1.053,1.335]^{P}$} & $1^{P}$ & 50.33 & $0.0577^{P}$ & $0.97^{P}$ & 165.60 & 15,960 \\
\hline & & $(13,24,30)$ & {$[0.926,0.973,1.000]^{N}$} & $0^{N}$ & & $0.0137^{N}$ & $0.98^{N}$ & & \\
\hline & & & {$[0.426,0.516,0.537]^{L}$} & $0^{L}$ & & $0.0034^{L}$ & $0.99^{L}$ & & \\
\hline & & & $(0.800,0.700,1.100)^{P}$ & & & & & & \\
\hline & & & $(0.300,0.400,1.000)^{N}$ & & & & & & \\
\hline & & & $(0.800,0.200,0.400)^{L}$ & & & & & & \\
\hline
\end{tabular}


TABLE V

SimUlation Results ObTAINED By CMSO FOR STUdy SyStem-II

\begin{tabular}{|c|c|c|c|c|c|c|c|c|}
\hline Case & $\begin{array}{l}\text { Optimal } \\
{[\text { DG]/(SC) }} \\
\text { nodes }\end{array}$ & Optimal [DG]/(SC) Sizes (MW/MVAr) & $\begin{array}{l}\text { OLTC } \\
\text { Tap }\end{array}$ & $\begin{array}{l}\text { DER } \\
\text { Pen. } \%\end{array}$ & $\begin{array}{l}\text { Power } \\
\text { Loss } \\
\text { (MW) }\end{array}$ & $V_{\min }$ & $\begin{array}{l}\text { AEL } \\
\text { (MWh) }\end{array}$ & $\begin{array}{l}\text { Cost of } \\
\text { AEL } \\
\text { (USD) }\end{array}$ \\
\hline \multirow{3}{*}{ I } & \multirow[t]{3}{*}{-} & \multirow[t]{3}{*}{ NA } & \multirow[t]{3}{*}{-} & \multirow[t]{3}{*}{00.00} & $3.7996^{P}$ & $0.77^{P}$ & \multirow[t]{3}{*}{13121.60} & \multirow[t]{3}{*}{1208,225} \\
\hline & & & & & $1.2981^{N}$ & $0.87^{N}$ & & \\
\hline & & & & & $0.2971^{L}$ & $0.94^{L}$ & & \\
\hline \multirow{3}{*}{ II } & \multirow{3}{*}{$\begin{array}{l}{[33,38,52,71} \\
80,96,109]\end{array}$} & {$[2.910,3.225,2.677,4.414,2.276,2.159,4.642]^{P}$} & \multirow[t]{3}{*}{ NA } & \multirow[t]{3}{*}{49.10} & $1.4622^{P}$ & $0.93^{P}$ & \multirow[t]{3}{*}{5241.30} & \multirow[t]{3}{*}{478,229} \\
\hline & & {$[2.147,2.414,1.741,2.808,2.023,1.627,3.118]^{N}$} & & & $0.5382^{N}$ & $0.96^{N}$ & & \\
\hline & & {$[1.035,1.249,0.820,1.356,1.031,0.811,1.527]^{L}$} & & & $0.1301^{L}$ & $0.98^{L}$ & & \\
\hline \multirow{3}{*}{ III } & \multirow{3}{*}{$\begin{array}{l}{[8,41,50,74,} \\
81,96,109]\end{array}$} & {$[0.444,2.521,3.807,3.176,2.261,2.516,4.567]^{P}$} & $4^{P}$ & 42.46 & $1.4225^{P}$ & $0.92^{P}$ & \multirow[t]{3}{*}{5179.55} & \multirow[t]{3}{*}{458,190} \\
\hline & & {$[0.189,1.805,2.781,2.541,1.844,1.663,3.124]^{N}$} & $2^{N}$ & & $0.5300^{N}$ & $0.97^{N}$ & & \\
\hline & & {$[0.189,0.916,1.368,1.204,0.909,0.855,1.521]^{L}$} & $1^{L}$ & & $0.1290^{L}$ & $0.98^{L}$ & & \\
\hline \multirow{6}{*}{ IV } & \multirow{6}{*}{$\begin{array}{l}{[34,42,52,59,} \\
73,81,110] \\
(34,50,72,85 \\
91,107,109)\end{array}$} & {$[2.822,1.866,1.940,0.742,3.870,1.629,2.928]^{P}$} & NA & 47.97 & $0.6260^{P}$ & $0.94^{P}$ & \multirow[t]{6}{*}{2121.56} & \multirow[t]{6}{*}{196,141} \\
\hline & & {$[1.904,1.239,1.556,0.500,2.484,1.456,2.478]^{N}$} & & & & & & \\
\hline & & {$[0.893,0.610,0.791,0.366,1.285,0.929,1.375]^{L}$} & & & $0.2060^{N}$ & $0.96^{N}$ & & \\
\hline & & $(2.100,2.200,1.500,1.300,1.000,1.800,2.200)^{P}$ & & & & & & \\
\hline & & $(0.900,2.100,1.400,0.900,0.900,1.000,1.700)^{N}$ & & & $0.0495^{L}$ & $0.98^{L}$ & & \\
\hline & & $(0.700,0.900,0.800,0.400,0.500,0.800,0.600)^{L}$ & & & & & & \\
\hline \multirow{6}{*}{$\mathrm{V}$} & \multirow{6}{*}{$\begin{array}{l}{[34,42,52,59} \\
73,81,110] \\
(50,60,73,80 \\
91,108,111)\end{array}$} & {$[2.822,1.866,1.940,0.742,3.870,1.629,2.928]^{P}$} & $3^{P}$ & 44.89 & $0.6208^{P}$ & $0.95^{P}$ & \multirow[t]{6}{*}{2024.00} & \multirow[t]{6}{*}{188,944} \\
\hline & & {$[1.904,1.239,1.556,0.500,2.484,1.456,2.478]^{N}$} & & & & & & \\
\hline & & {$[0.893,0.610,0.791,0.366,1.285,0.929,1.375]^{L}$} & $2^{N}$ & & $0.1906^{N}$ & $0.97^{N}$ & & \\
\hline & & $(2.500,1.100,1.900,2.500,1.900,1.200,1.800)^{P}$ & & & & & & \\
\hline & & $(2.300,0.800,1.500,1.700,0.800,0.900,1.500)^{N}$ & $1^{L}$ & & $0.0456^{L}$ & $0.97^{L}$ & & \\
\hline & & $(1.200,0.300,0.800,0.800,0.600,0.300,0.900)^{L}$ & & & & & & \\
\hline
\end{tabular}

to Case-II. However, slightly higher voltage deviation has been observed during peak load hours due to low DG penetration and larger size of the system.

In Case-IV, the optimal allocation of DGs and SCs is simultaneously determined in order to provide the VAr support into the system. The optimal solution shows that the consideration of SCs further increases the integration benefits of DGs. It also helps the utilities to reduce initial investment of DGs, since SCs are cheaper than DGs. Further, the optimal allocation of DGs and SCs, and OLTC tap settings is simultaneously determined in Case-V. It shows that consideration of OLTC while optimally allocating DERs significantly improves the system performance in terms of AEL cost reduction and improved voltage profile at smaller DG penetration.

In overall consideration, the proposed DER allocation model provides larger benefits to utility, consumers, and DER owners while utilizing the existing distribution system resources from small to large distribution networks. However, it is very difficult to improve the performance of large-scale distribution systems. The proposed model also minimizes the coordination issues in daily operations as the effects of existing VRs are already considered while planning new DERs in the system.

\section{CONCLUSION}

The paper introduces a powerful optimization technique, i.e., MSO, to solve real-life complex DER integration problem, in conjunction with existing OLTC in distribution systems. MSO has well-balanced exploitation and exploration capabilities to search local and global solutions. Despite balanced mechanism, this technique shows inherent tendencies of local trapping. Therefore, some corrections have also been suggested.
The proposed meliorations help the method to avoid the local trappings, as validated by comparing its performance with the standard variant. It has been observed that the suggested corrections improved the exploration capability of the method, i.e., global solution. The objective of the proposed DER allocation model is to minimize the cost of AEL and node voltage deviation of distribution systems simultaneously. The case study is demonstrated that consideration of OLTC-based VR generates more techno-economic benefits at lesser DG penetration. The proposed model effectively utilizes the existing system resources.

It is observed that the method has many parameters to tune. The values of these quantities are generally varying with problem complexity. The parameter tuning can be considered as one of the limitations of this method. In future, some work can be done in order to effectively reduce the number of parameters without changing the inherent mechanism of the method or a parameter tuning algorithm may be suggested. Furthermore, the proposed DER integration model may be explored for other existing VRs and renewables.

\section{REFERENCES}

[1] T. Ackermann, G. Andersson, and L. Söder, "Distributed generation: A definition," Electr. Power Syst. Res., vol. 57, no. 3, pp. 195-204, 2001.

[2] W. El-Khattam and M. M. A. Salama, "Distribution system planning using distributed generation," in Proc. Can. Conf. Electr. Comput. Eng. Toward a Caring Humane Technol (Cat. no. 03CH37436), vol. 1, May 2003, pp. 579582.

[3] P. S. Georgilakis and N. D. Hatziargyriou, "Optimal distributed generation placement in power distribution networks: Models, methods, and future research," IEEE Trans. Power Syst., vol. 28, no. 3, pp. 3420-3428, Aug. 2013. 
[4] C. L. Borges and D. M. Falcao, "Optimal distributed generation allocation for reliability, losses, and voltage improvement," Int. J. Electr. Power Energy Syst., vol. 28, no. 6, pp. 413-420, 2006.

[5] N. K. Meena, A. Swarnkar, N. Gupta, and K. R. Niazi, "Optimal integration of DERs in coordination with existing VRs in distribution networks," IET Gener. Transmiss. Distribution, vol. 12, no. 11, pp. 2520-2529, Jun. 2018.

[6] N. Kanwar, N. Gupta, K. Niazi, and A. Swarnkar, "Simultaneous allocation of distributed resources using improved teaching learning based optimization," Energy Convers. Manage., vol. 103, pp. 387-400, 2015.

[7] A. M. Imran, M. Kowsalya, and D. Kothari, "A novel integration technique for optimal network reconfiguration and distributed generation placement in power distribution networks," Int. J. Elect. Power Energy Syst., vol. 63, pp. 461-472, 2014.

[8] S. Sultana and P. K. Roy, "Multi-objective quasi-oppositional teaching learning based optimization for optimal location of distributed generator in radial distribution systems," Int. J. Elect. Power Energy Syst., vol. 63, pp. 534-545, 2014.

[9] D. R. Prabha and T. Jayabarathi, "Optimal placement and sizing of multiple distributed generating units in distribution networks by invasive weed optimization algorithm," Ain Shams Eng. J., vol. 7, no. 2, pp. 683-694, 2016.

[10] H. Hamedi and M. Gandomkar, "A straightforward approach to minimizing unsupplied energy and power loss through DG placement and evaluating power quality in relation to load variations over time," Int. J. Elect. Power Energy Syst., vol. 35, no. 1, pp. 93-96, 2012.

[11] N. K. Meena, S. Parashar, A. Swarnkar, N. Gupta, and K. R. Niazi, "Improved elephant herding optimization for multiobjective DER accommodation in distribution systems," IEEE Trans. Ind. Inform., vol. 14, no. 3, pp. 1029-1039, Mar. 2018.

[12] N. K. Meena, A. Swarnkar, N. Gupta, and K. R. Niazi, "Multi-objective Taguchi approach for optimal DG integration in distribution systems," IET Gener. Transmiss. Distribution, vol. 11, no. 9, pp. 2418-2428, 2017.

[13] M. Moradi and M. Abedini, "A combination of genetic algorithm and particle swarm optimization for optimal DG location and sizing in distribution systems," Int. J. Electr. Power Energy Syst., vol. 34, no. 1, pp. 66-74, 2012.

[14] A. S. A. Awad, T. H. M. El-Fouly, and M. M. A. Salama, "Optimal distributed generation allocation and load shedding for improving distribution system reliability," Electr. Power Components Syst., vol. 42, no. 6, pp. 576-584, 2014.

[15] S. K. Injeti and N. P. Kumar, "A novel approach to identify optimal access point and capacity of multiple DGs in a small, medium, and large scale radial distribution systems," Int. J. Electr. Power Energy Syst., vol. 45, no. 1, pp. 142-151, 2013.

[16] D. Q. Hung and N. Mithulananthan, "Multiple distributed generator placement in primary distribution networks for loss reduction," IEEE Trans. Ind. Electron., vol. 60, no. 4, pp. 1700-1708, Apr. 2013.

[17] S. N. G. Naik, D. K. Khatod, and M. P. Sharma, "Analytical approach for optimal siting and sizing of distributed generation in radial distribution networks," IET Gener. Transmiss. Distribution, vol. 9, no. 3, pp. 209-220, Feb. 2015.

[18] K. Nekooei, M. M. Farsangi, H. Nezamabadi-Pour, and K. Y. Lee, “An improved multi-objective harmony search for optimal placement of DGs in distribution systems," IEEE Trans. Smart Grid, vol. 4, no. 1, pp. 557-567, Mar. 2013.

[19] A. Askarzadeh, "Capacitor placement in distribution systems for power loss reduction and voltage improvement: A new methodology," IET Gener. Transmiss. Distribution, vol. 10, no. 14, pp. 3631-3638, Apr. 2016.

[20] K. Muthukumar and S. Jayalalitha, "Optimal placement and sizing of distributed generators and shunt capacitors for power loss minimization in radial distribution networks using hybrid heuristic search optimization technique," Int. J. Elect. Power Energy Syst., vol. 78, pp. 299-319, 2016.

[21] A. A. A. El-Ela, R. A. El-Sehiemy, and A. S. Abbas, "Optimal placement and sizing of distributed generation and capacitor banks in distribution systems using water cycle algorithm," IEEE Syst. J., no. 99, pp. 1-8, Dec. 2018.

[22] R. S. Rao, K. Ravindra, K. Satish, and S. Narasimham, "Power loss minimization in distribution system using network reconfiguration in the presence of distributed generation," IEEE Trans. Power Syst., vol. 28, no. 1, pp. 317-325, Feb. 2013.

[23] S. Ganguly and D. Samajpati, "Distributed generation allocation with on-load tap changer on radial distribution networks using adaptive genetic algorithm," Appl. Soft Comput., vol. 59, pp. 45-67, 2017.
[24] D. Q. Hung, N. Mithulananthan, and R. C. Bansal, "Analytical expressions for DG allocation in primary distribution networks," IEEE Trans. Energy Convers., vol. 25, no. 3, pp. 814-820, Sep. 2010.

[25] I. A. Quadri, S. Bhowmick, and D. Joshi, "A comprehensive technique for optimal allocation of distributed energy resources in radial distribution systems," Appl. Energy, vol. 211, pp. 1245-1260, 2018.

[26] G.-G. Wang, "Moth search algorithm: A bio-inspired metaheuristic algorithm for global optimization problems," Memetic Comput., vol. 10, pp. 151-164, 2018.

[27] N. K. Meena, A. Swarnkar, N. Gupta, and K. R. Niazi, "A Taguchi-based approach for optimal placement of distributed generations for power loss minimization in distribution system," in Proc. IEEE Power Energy Soc. General Meeting, Jul. 2015, pp. 1-5.

[28] H. Saadat, Power System Analysis, vol. 232. Singapore: WCB/McGrawHill, 1999.

[29] K. Bryan and Y. Shibberu, Penalty Functions and Constrained Optimization. Terre Haute, IN 47803, USA: Dept. of Mathematics, Rose-Hulman Institute of Technology, 2005. [Online]. Available: https://www.rosehulman.edu/ bryan/lottamath/penalty.pdf

[30] Y. Li, Y. Sun, B. Li, and Z. Xu, "Penalty function-based method for obtaining a reliability indicator of gravity dam stability," Comput. Geotechnics, vol. 81, pp. 19-25, 2017.

[31] B. Tessema and G. G. Yen, "A self adaptive penalty function based algorithm for constrained optimization," in Proc. Evol. Comput. CEC IEEE Congr. IEEE, 2006, pp. 246-253.

[32] M.E. Baran and F. F. Wu, "Network reconfiguration in distribution systems for loss reduction and load balancing," IEEE Trans. Power Del., vol. 4, no. 2, pp. 1401-1407, Apr. 1989.

[33] D. Zhang, Z. Fu, and L. Zhang, "An improved TS algorithm for lossminimum reconfiguration in large-scale distribution systems," Electr. Power Syst. Res., vol. 77, no. 5, pp. 68-694, 2007.

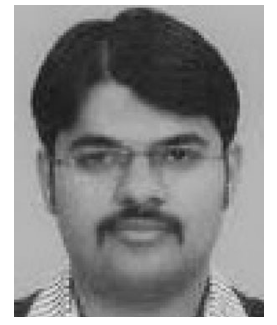

Pushpendra Singh (S'19) was born in Rajasthan, India. He received the M.Tech. degree in power systems from the Malaviya National Institute of Technology (MNIT) Jaipur, Jaipur, India, in 2011.

$\mathrm{He}$ is currently an Assistant Professor with the Department of Electrical Engineering, Govt. Women Engineering College Ajmer, Ajmer, India and working toward the Ph.D. degree at the Rajasthan Technical University Kota, Kota, India. His Ph.D. research is focused on DER planning, optimization techniques, and electrical energy storage.

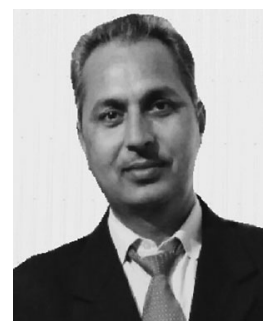

S. K. Bishnoi was born in Jodhpur, India in 1967. He received the M.E. degree in control systems from the Mugneeram Bangur Memorial (MBM) Engineering College Jodhpur, Jodhpur, India, in 2000, and the Ph.D. degree from Malaviya National Institute of Technology (MNIT) Jaipur, Jaipur, India, in 2012.

$\mathrm{He}$ is currently an Associate Professor with the Department of ECE, Govt. Engineering College, Bikaner, India. His research interests include control $\&$ drives, AI-techniques, and DER planning.

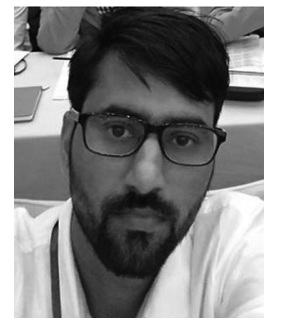

Nand K. Meena (S'13-M'18) received the B.Tech. and the M. Tech. (dual) degrees from the Indian Institute of Technology (IIT) Kanpur, Kanpur, India, in 2011, and the Ph.D. degree from the MNIT Jaipur, Jaipur, India, in 2018, all in electrical engineering.

He is currently a Research Fellow with the School of Engineering and Applied Science, Aston University, Birmingham, U.K. His current research interests include planning \& operational management of active distribution systems, microgrids, electric vehicles, energy storage, and application of AI-techniques 